\title{
EBV-positive mucocutaneous ulcer of the oral cavity associated with HIV/AIDS
}

Belinda Bunn, BDS, MDent, FC Path(SA) Oral Path, ${ }^{\text {a }}$ Willie van Heerden, BChD, MChD, FC Path(SA) Oral Path, PhD, DSc. ${ }^{\mathrm{a}}$

${ }^{\mathrm{a}}$ Department of Oral Pathology and Oral Biology, School of Dentistry, Faculty of Health Sciences, University of Pretoria, South Africa.

Address for correspondence: Professor WFP van Heerden, Department of Oral Pathology and Oral Biology, PO Box 1266, Pretoria, South Africa.

Tel: +27123192320; Fax: +27123212225; e-mail: willie.vanheerden@up.ac.za

The authors declare that there are no conflicts of interest.

Disclosure: This work will be presented as a poster presentation by the same authors at the $27^{\text {th }}$ European Congress of Pathology to be held in Belgrade, Serbia 5-9 September, 2015.

Word count abstract: 150 words

Word count manuscript (body text and figure legends): 3544 words

References: 20

Figures: 11

Tables: 1 


\begin{abstract}
Objective: To present two cases of Epstein-Barr virus (EBV)-positive mucocutaneous ulcer of the oral mucosa in association with human immunodeficiency virus infection.

Study design: Two recently diagnosed cases of EBV-positive mucocutaneous ulcer of the oral mucosa in HIV-positive patients were reviewed with regards to their clinical, histomorphological and immunophenotypic features.
\end{abstract}

Results: Both cases presented clinically as well circumscribed ulcers which were histomorphologically characterized by dense superficial polymorphous inflammatory infiltrates. The infiltrates comprised cells with a predominant B-cell phenotype which ranged in size from small to intermediate with occasional large immunoblastic forms. Some of the larger B-cells had a Reed-Sternberg-like morphology. The B-cells were positive for CD20 and co-expressed CD30 and to a lesser extent CD15. EBER positivity was detected in most of the B-cells.

Conclusion: EBV-positive mucocutaneous ulcer represents an unusual form of lymphoproliferative disorder associated with immune suppression. It should be distinguished from other forms of HIV-associated oral ulceration.

\title{
STATEMENT OF CLINICAL RELEVANCE
}

EBV-positive mucocutaneous ulcer should be considered in the differential diagnosis of HIVassociated oral ulceration. Its unique clinical, histomorphological and immunophenotypic features should allow for its distinction in this setting, the diagnosis of which necessitates a conservative immune modulating therapeutic approach. 


\section{INTRODUCTION}

Oral ulceration is a frequent clinical manifestation and complication of human immunodeficiency virus (HIV) infection with an estimated prevalence of 10 to $15 \%$ within this patient group. The incidence of oral ulceration increases in end stage disease (AIDS acquired immunodeficiency syndrome) and with decreased numbers of CD4 ${ }^{+}$T-cells. ${ }^{1,2}$ Oral ulceration may, however, be seen at any stage of HIV infection. The diagnosis of HIV/AIDS associated oral ulceration is complex due to the multitude of potential etiologies and known causes. Ulceration in these patients, irrespective of the cause, is more aggressive, painful and severely debilitating. These ulcers tend to have a protracted clinical course and are frequently refractory to therapy. $1,3,4$

HIV/AIDS associated oral ulcers are predominantly infectious or neoplastic in origin., ${ }^{1,4}$ Non-infectious, non-neoplastic forms of ulceration with unusual clinical presentations and where the etiopathogenesis is unknown, are grouped together as "ulceration not otherwise specified (NOS)". 5 This group of ulcers includes those with recurrent aphthous-type features. The rapidly progressive opportunistic infectious process of necrotizing ulcerative stomatitis (NUS) shares many of the atypical clinical and non-specific histological features of the ulcers in this category. ${ }^{1,6}$ The range of polychemotherapy and multiple drug regimens to which HIV-positive patients are exposed for the treatment of HIV infection itself as well as the range of infectious and neoplastic diseases they suffer from, significantly increases the risk of potential drug interactions and cytotoxic tissue effects. This may result in iatrogenic ulceration. $^{7}$

Epstein-Barr virus-positive mucocutaneous ulcer (EBV MCU) is a recently documented entity that occurs in associated with various forms of immunosuppression. Lesions have been described in the oral cavity, gastrointestinal tract and $\operatorname{skin}^{8-10}$. They represent a unique, 
indolent form of EBV-driven B-cell lymphoproliferative disorder (LPD) which present as isolated, well-delineated ulcers that respond well to conservative therapeutic intervention. The previously documented cases have occurred in patients on immunosuppressive medication and in the elderly with age-related immune senescence. ${ }^{9,11}$ EBV LPDs are known to occur in the setting of HIV/AIDS; however, their presentation and response to treatment are not well documented. LPDs in these patients most closely resemble the polymorphic lymphoid proliferations described in post-transplant patients. ${ }^{12,13} \mathrm{EBV} \mathrm{MCU}$ to the best of our knowledge has not yet been described in immunosuppression due to HIV infection.

Confounding both the clinical and histopathological presentation of HIV-associated oral ulceration is the frequent occurrence of multiple co-existent pathologies in the same biopsy specimens. This is often characterized by multiple infectious processes occurring side by side with neoplastic or non-neoplastic disease. It is thus essential that clinicians obtain comprehensive clinical and medical histories in these patients and that chronic ulcers be biopsied in order to establish the most accurate diagnosis to facilitate appropriate management. ${ }^{1,4}$

We have recently encountered two cases of oral ulceration in HIV-positive patients, which have the clinical, histomorphological, and immunophenotypic features of EBV MCU. The aim of this study is to present this unusual form of oral ulceration in association with HIV infection for the first time. 


\section{CASE PRESENTATIONS}

\section{Case one}

A 54-year old HIV-positive male patient presented with a three-month history of an isolated area of painful ulceration involving the right soft palate. The area of ulceration extended to involve the tonsillar pillar and right maxillary retromolar area extending into the soft palate measuring $4 \mathrm{~cm}$ in largest diameter (Figure 1). Furthermore, the patient had marked trismus

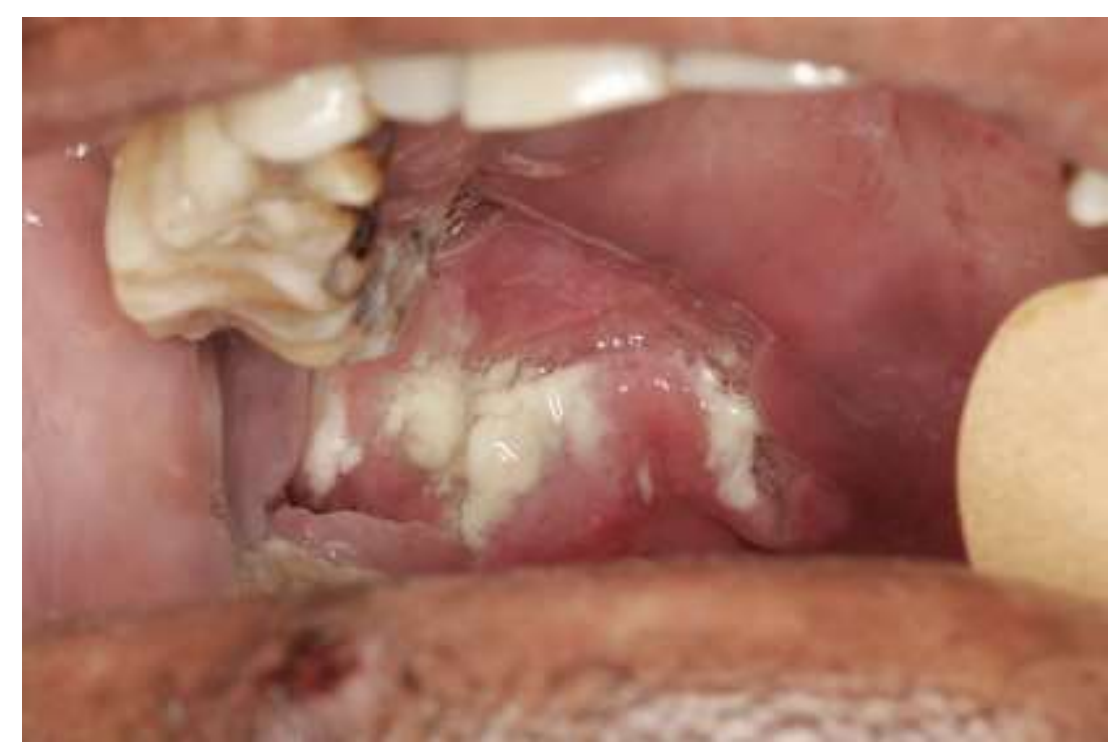

Fig. 1. Intra-oral ulceration of the right soft palate, tonsillar and maxillary retromolar area in a 54 year old HIVpositive male patient (case one).

and dysphagia. He had notable weight loss and cervical lymphadenopathy on clinical examination. The patient had been on antiretroviral therapy (Tenovir 300mg daily, Lamivudine 300mg daily and Nevirapine 200mg twide daily) for one year at the time of clinical presentation, although he openly admitted to non-compliance. Two previous incisional biopsies had shown features of non-specific acute-on-chronic ulceration. A third incisional biopsy obtained from the junction of the hard and soft palate was submitted for histopathological assessment. The clinical differential diagnosis included squamous cell carcinoma and histoplasmosis. 


\section{Case two}

A 36-year old HIV-positive female patient presented with a two week history of trismus. Intra-oral examination showed an area of ulceration of the left soft palate measuring $1.5 \mathrm{~cm}$ in largest diameter (Figure 2). The patient had a $\mathrm{CD}^{+}{ }^{+}$-cell count of $12 / \mu 1$ and a viral load of 219837 copies/ml. She was not on any antiretroviral therapy at presentation. Squamous cell carcinoma or a salivary gland malignancy was clinically considered. An incisional biopsy was submitted for histopathology.

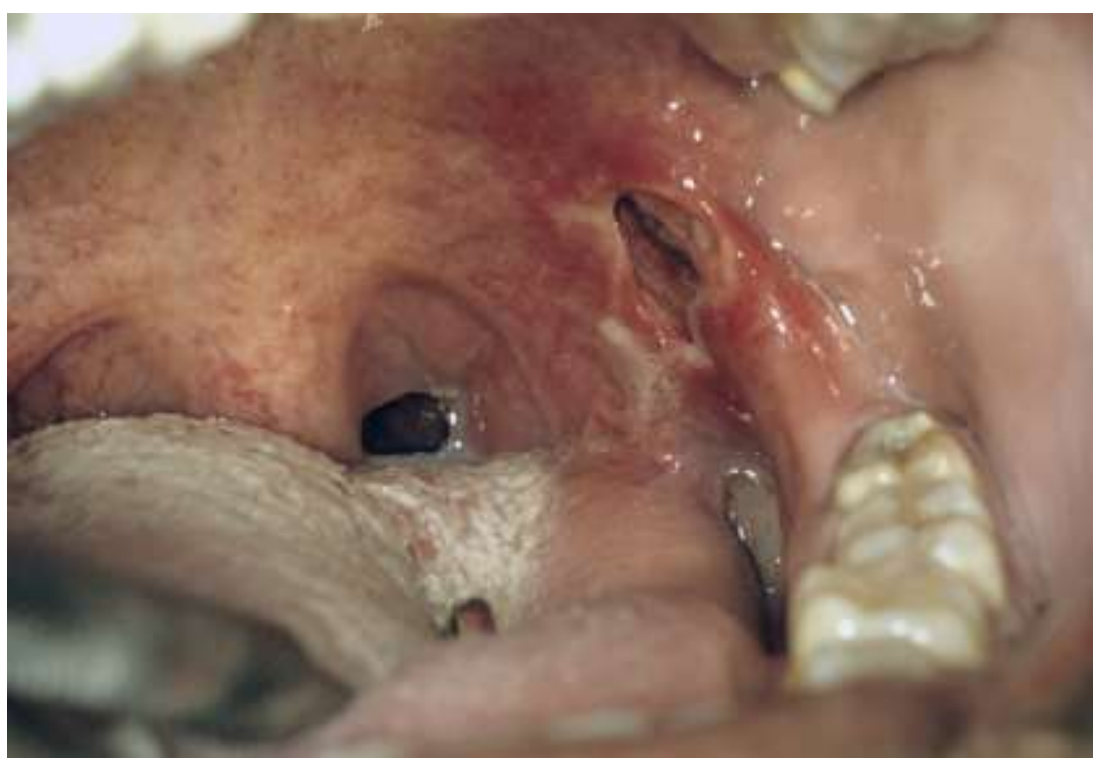

Fig. 2. Area of ulceration involving the left soft palate and tonsillar pillar in a 36-year old HIV-positive female patient (case two).

\section{MATERIALS AND METHODS}

The histomorphological features noted in the two cases presented above were reviewed. The clinical details in each case were obtained from the histological request forms, patient records and through discussion with the clinicians in each case. The incisional biopsies were received in $10 \%$ neutral buffered formalin and stained by means of hematoxylin and eosin for routine light microscopic examination. The criteria for inclusion of the cases presented above were the presence of an isolated ulcerated lesion within the oral cavity in which an intense 
polymorphous infiltrate within the superficial submucosa and lamina propria was identified. The lesions had to have the clinical, morphological and immunophenotypic features which have been previously documented in the literature. ${ }^{9}$

\section{Immunohistochemistry}

Immunohistochemical staining (IHC) using mouse monoclonal antibodies to CD3, CD20, CD30, CD15, CD68 and Ki-67 was performed on both cases (Table 1). Three micrometer formalin fixed paraffin embedded (FFPE) tissue sections were de-paraffinized and hydrated followed by heat induced epitope retrieval (HIER) with TRIS/EDTA buffer $\mathrm{pH} 9$ for all above mentioned antibodies except CMV on which enzyme digestion was performed for 6 minutes at room temperature with a $0.05 \%$ pronase solution in $\mathrm{Tris} / \mathrm{HCl} \mathrm{pH} 7.2$ buffer. After incubation with the primary antibody, positivity was detected by the Novocastra Novolink Polymer Detection kit (Leica Biosystems, Newcastle Upon Tyne, UK) for ab's CD3, CD20 and Ki67 whilst antibodies CD30, CD15, CD68 and CMV were detected with Dako EnvisionTM + (Dako, Glostrup, Denmark) detection system. Positive and negative controls were used for verification of the staining in each case.

\section{EBV in situ hybridization}

Tissue sections three micrometer in thickness were cut from the FFPE tissue blocks and baked at $58^{\circ} \mathrm{C}$. EBV mRNA in-situ-hybridization was performed using proprietary reagents (Inform EBER DNP RNA probe, Roche Diagnostics, Mannheim Germany) on a Benchmark GX automated system (Ventana, Tucson, AZ) and visualized using the Ventana ISH/iView Blue detection kit and Red counterstain according to the manufacturer's instructions. The INFORM EBER probe is a fluorescein-labelled oligonucleotide probe that detects early RNA transcripts EBER-1 and EBER-2. Positive and negative controls were used for verification of staining. 


\section{RESULTS}

\section{Microscopic features}

Histological examination of the hematoxylin and eosin stained tissue sections from both case one and two showed similar morphological features. Large areas of mucosal ulceration surfaced by a fibrinopurulent membrane were noted in both biopsy specimens. The adjacent hyperplastic stratified squamous epithelium showed features of inflammatory mediated cytological atypia. The ulcer bed comprised inflamed granulation tissue whilst a dense inflammatory infiltrate was present within the lamina propria and superficial submucosa in each case (Figure 3). The inflammatory infiltrate extended between minor salivary gland

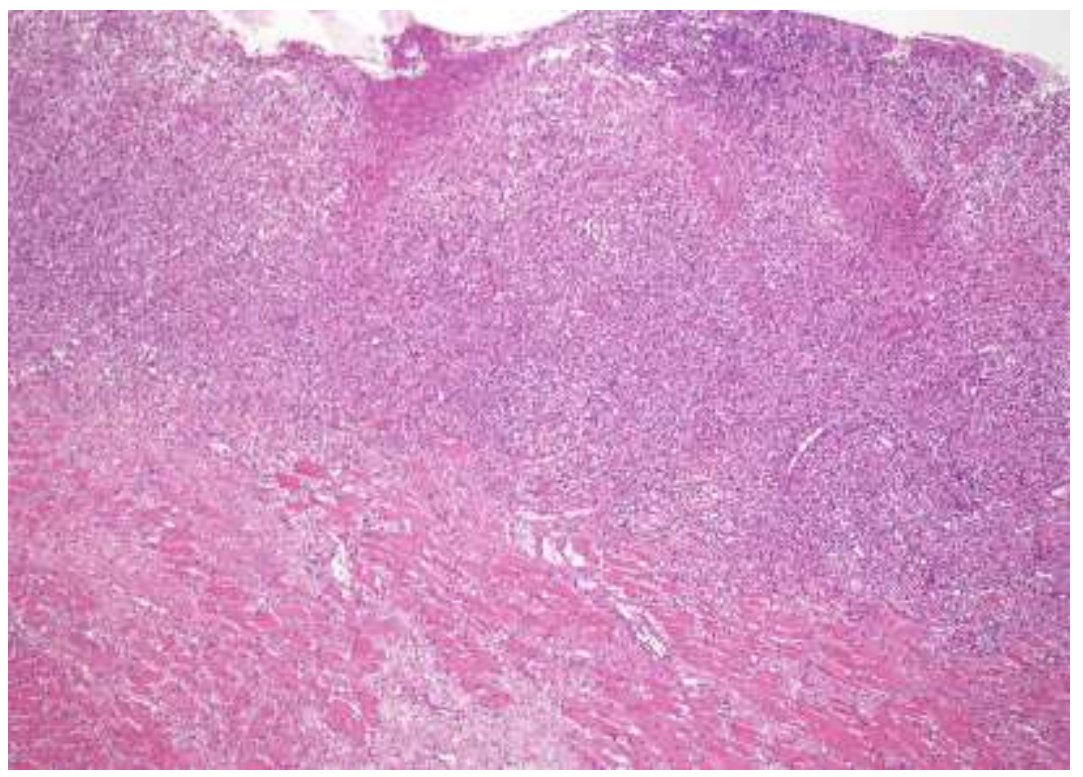

Fig. 3. Low power view of the ulcer obtained from the soft palate in case two in which the ulcer bed and superficially located hematolymphoid infiltrate are observed (hematoxylin-eosin, original magnification x20). A high-resolution version of this slide for use with the Virtual Microscope is available as eSlide: VM00706.

lobules in case one and into superficial skeletal muscle bundles in case two. The infiltrate was striking for its polymorphous population of inflammatory cells. The infiltrates in both cases comprised numerous small, non-descript lymphocytes, plasma cells, histiocytes and 
very occasional neutrophils and eosinophils. Intermediate to large lymphocytes with distinctly angulated nuclei were present (Figure 4).

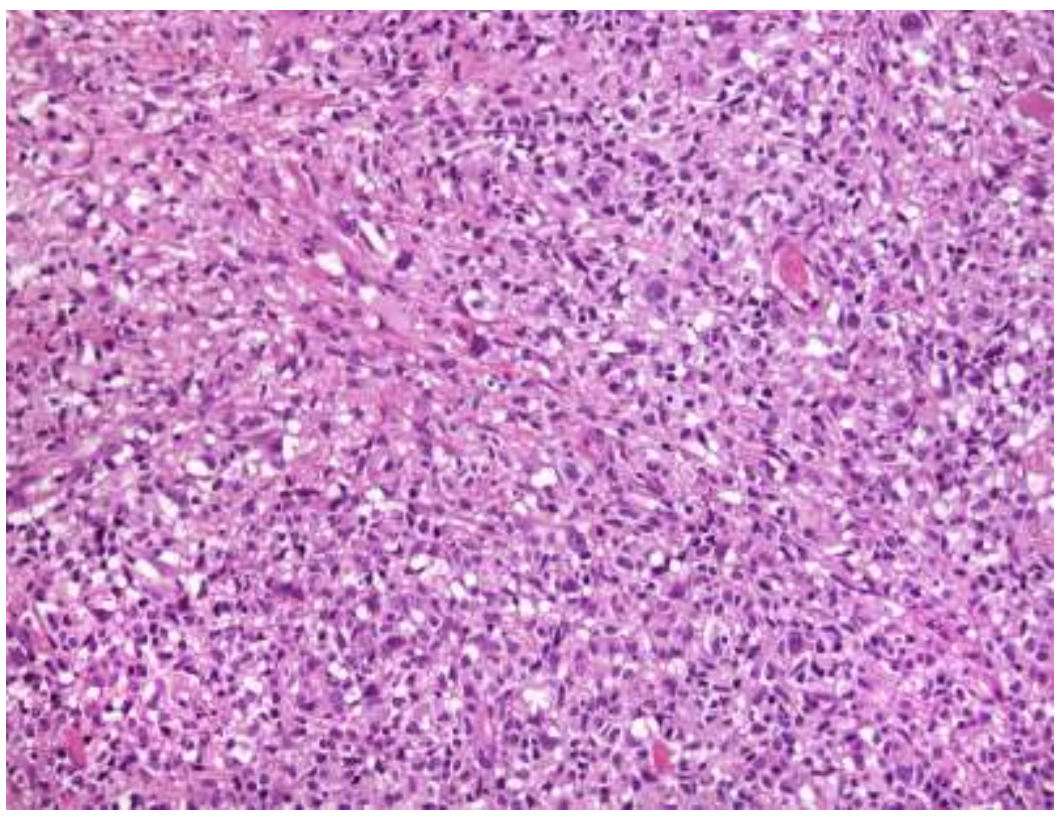

Fig. 4. The polymorphous nature of the inflammatory infiltrate is demonstrated in this microscopic image obtained from case two. The infiltrate contains small lymphocytes and occasional larger lymphocytes with distinctly angulated indented nuclei (hematoxylin-eosin, original magnification x100). A high-resolution version of this slide for use with the Virtual Microscope is available as eSlide: VM00706.

Large immunoblastic cells, each containing between 1-3 centrally located nuclei were scattered throughout. Some of the large atypical cells had a somewhat Reed-Sternberg-like (RS-like) morphology (Figure 5). The neutrophils in both cases were largely restricted to the area in the vicinity of the ulcer bed whilst the few eosinophils noted were present throughout. There was no evidence of granulomatous inflammation in any of the sections examined. Fungal elements were not noted within the infiltrates or within the superficial parakeratin layers of the adjacent intact epithelium. This was confirmed by negative results with special stains (PAS and Grocott's). The areas of ulceration were fairly well-circumscribed in both cases. The intralesional blood vessels showed no microscopic evidence of a vasculitis or of intraluminal microthrombus formation. Despite the worrisome morphology of the occasional 


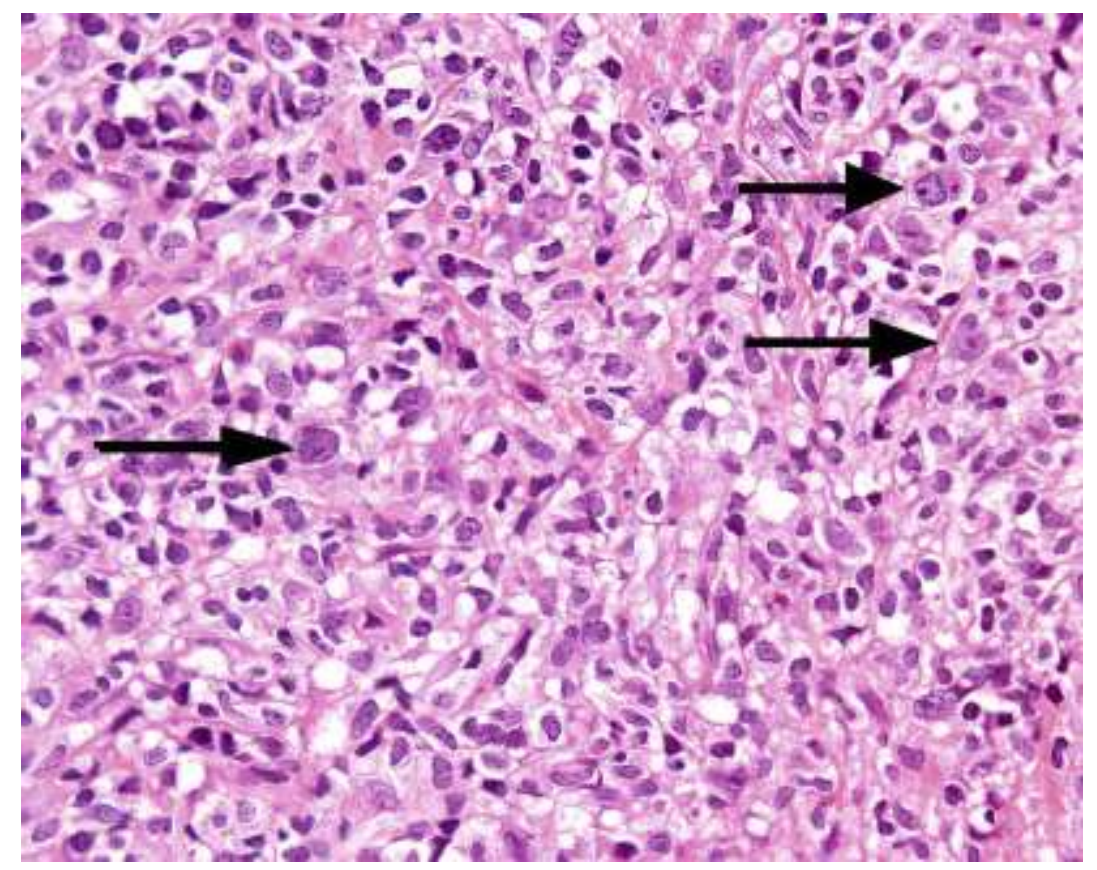

Fig. 5. This micrograph illustrates the range of lymphocytes including smaller cells, intermediate cells with angulated nuclei as well as large immunoblastic cells and occasional interspersed cells with RS-like morphology (arrows) (hematoxylin-eosin, original magnification x200 - case two). A high-resolution version of this slide for use with the Virtual Microscope is available as eSlide: VM00706.

immunoblastic and RS-like cells, the infiltrates did not appear to be overtly neoplastic largely due to their polymorphous nature.

\section{Immunohistochemical profile}

The polymorphous nature of the inflammatory infiltrates in both cases was confirmed by means of immunohistochemical staining. A large proportion of the small non-descript lymphocytes were positive for the T-cell marker CD3 (Figure 6). The B-cell marker CD20 stained many of the smaller lymphocytes as well as the intermediate cells with angulated nuclei. In addition, the larger immunoblastic cells as well as the RS-like cells were also positive for CD20 (Figure 7). Several admixed macrophages were highlighted by means of CD68 immunopositivity. The proliferative index marker Ki-67 showed nuclear signaling within 30 to $50 \%$ of the cells including many of the immunoblastic cells. CD30 positivity 


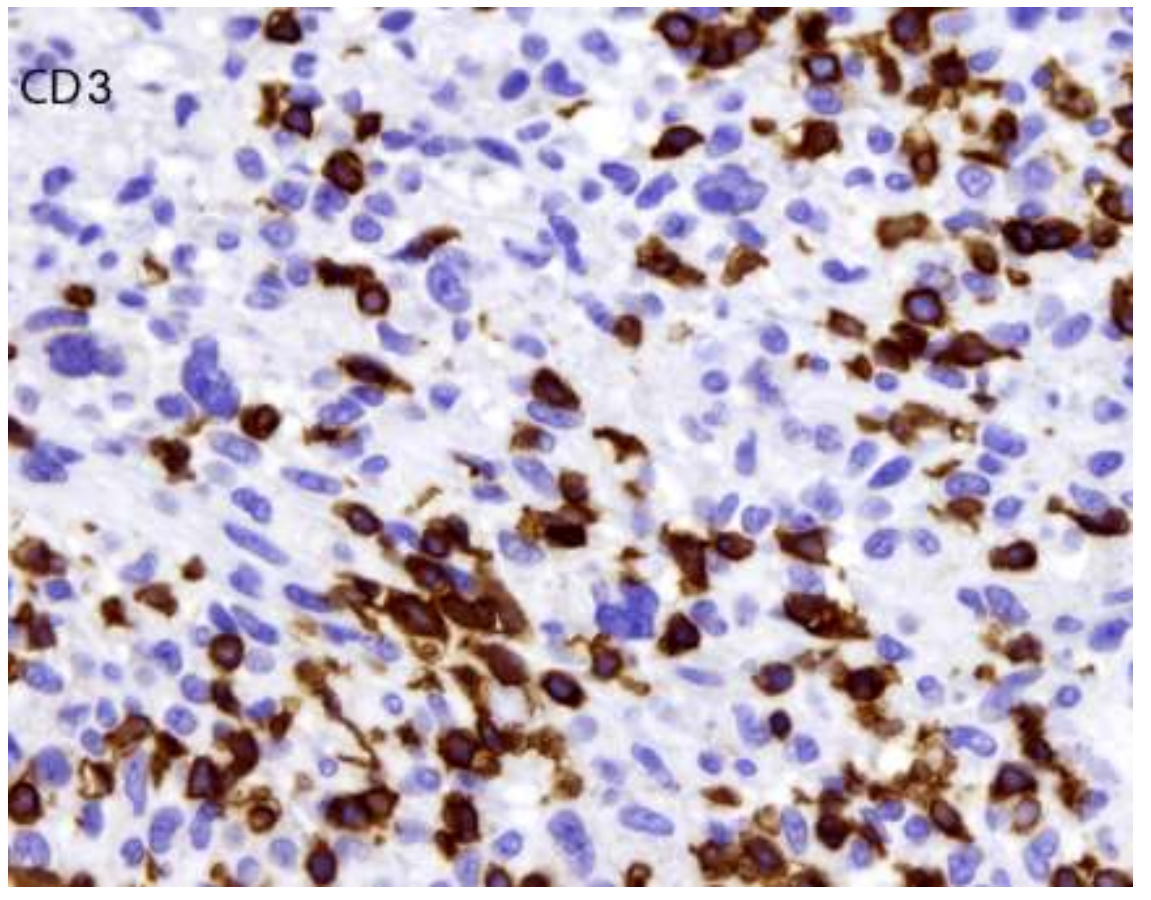

Fig. 6. Numerous background small T-cells are highlighted by means of CD3 (CD3, original magnification x200 - case two). A high-resolution version of this slide for use with the Virtual Microscope is available as eSlide: VM00707.

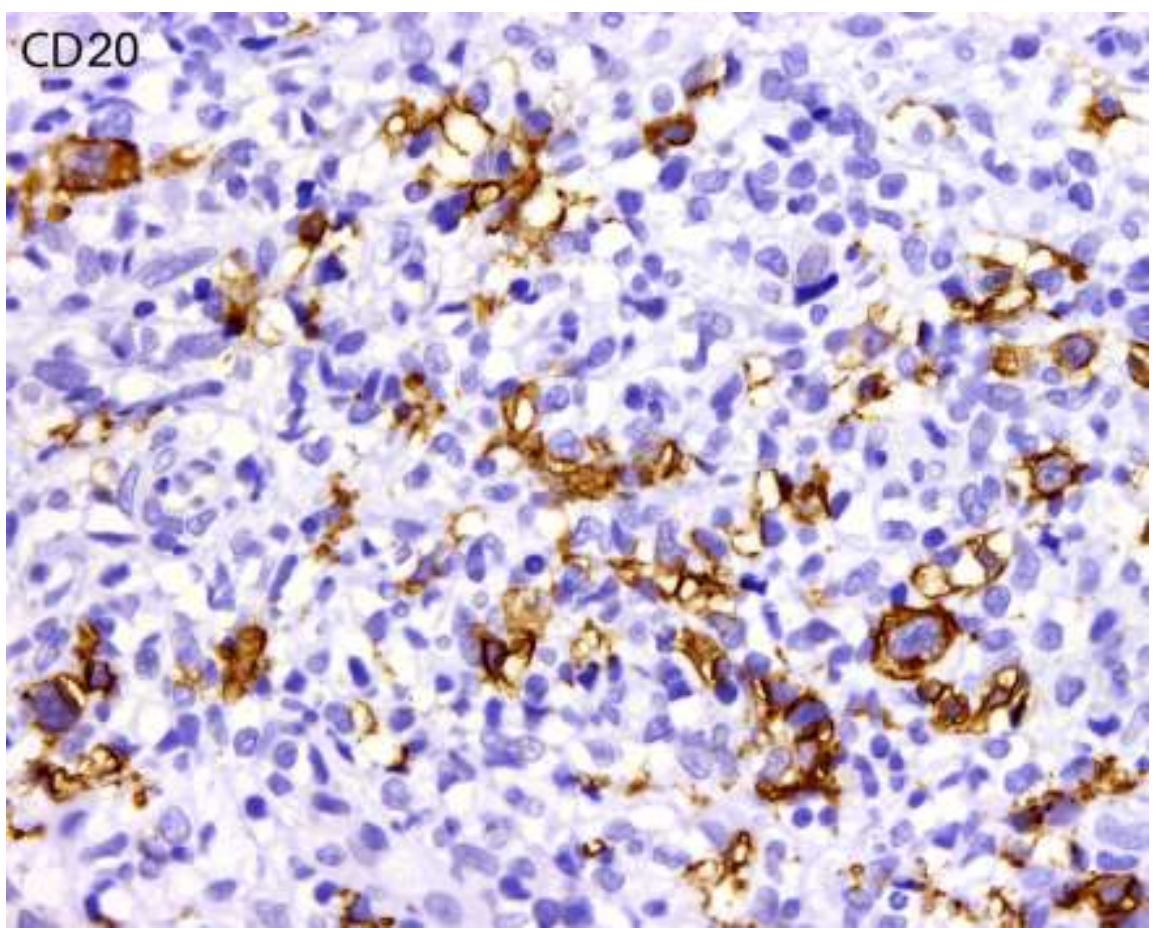

Fig. 7. CD20 staining shows a range of B-cells including some of the smaller lymphocytes, those with angulated nuclei and larger immunoblastic cells (CD20, original magnification x200 - case two). A highresolution version of this slide for use with the Virtual Microscope is available as eSlide: VM00708. 


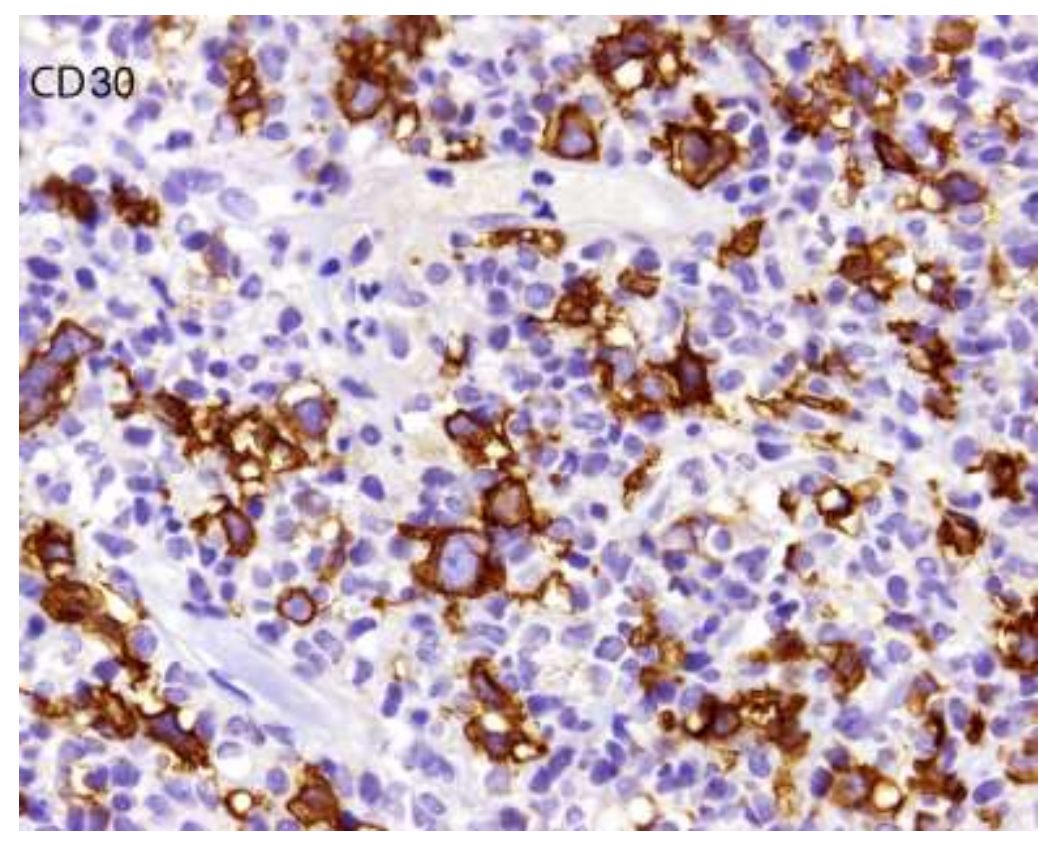

Fig. 8. A range of smaller lymphocytes, the intermediate cells with angulated nuclei as well as the immunoblastic and RS-like cells show immunoreactivity to CD30 as demonstrated in this micrograph (CD30, original magnification x200 - case two). A high-resolution version of this slide for use with the Virtual

Microscope is available as eSlide: VM00709.

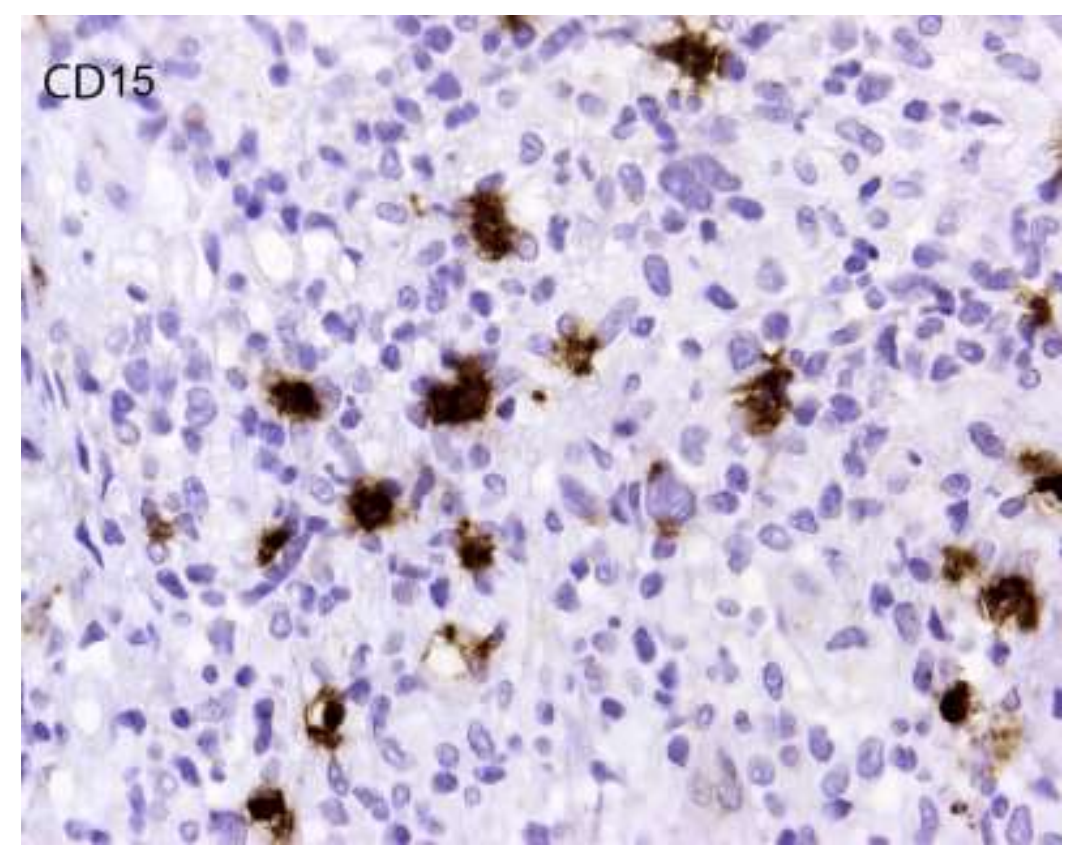

Fig. 9. CD15 expression within the immunoblastic and RS-like cells is demonstrated in this micrograph showing a slight paranuclear dot-like pattern of staining (CD15, original magnification x200 - case two). A high-resolution version of this slide for use with the Virtual Microscope is available as eSlide: VM00710. 
was detected in a large number of the smaller B-lymphocytes as well as in the larger immunoblastic and RS-like cells (Figure 8). CD15 immunopositivity was demonstrated in very occasional cells, predominantly the larger immunoblastic cells, often with a paranuclear dot-like accentuation (Figure 9).

\section{EBV in situ hybridization}

EBER staining was detected in several of the smaller lymphocytes as well as in many of the larger B-cells including the immunoblastic and RS-like cells (Figures $10 \& 11$ ).

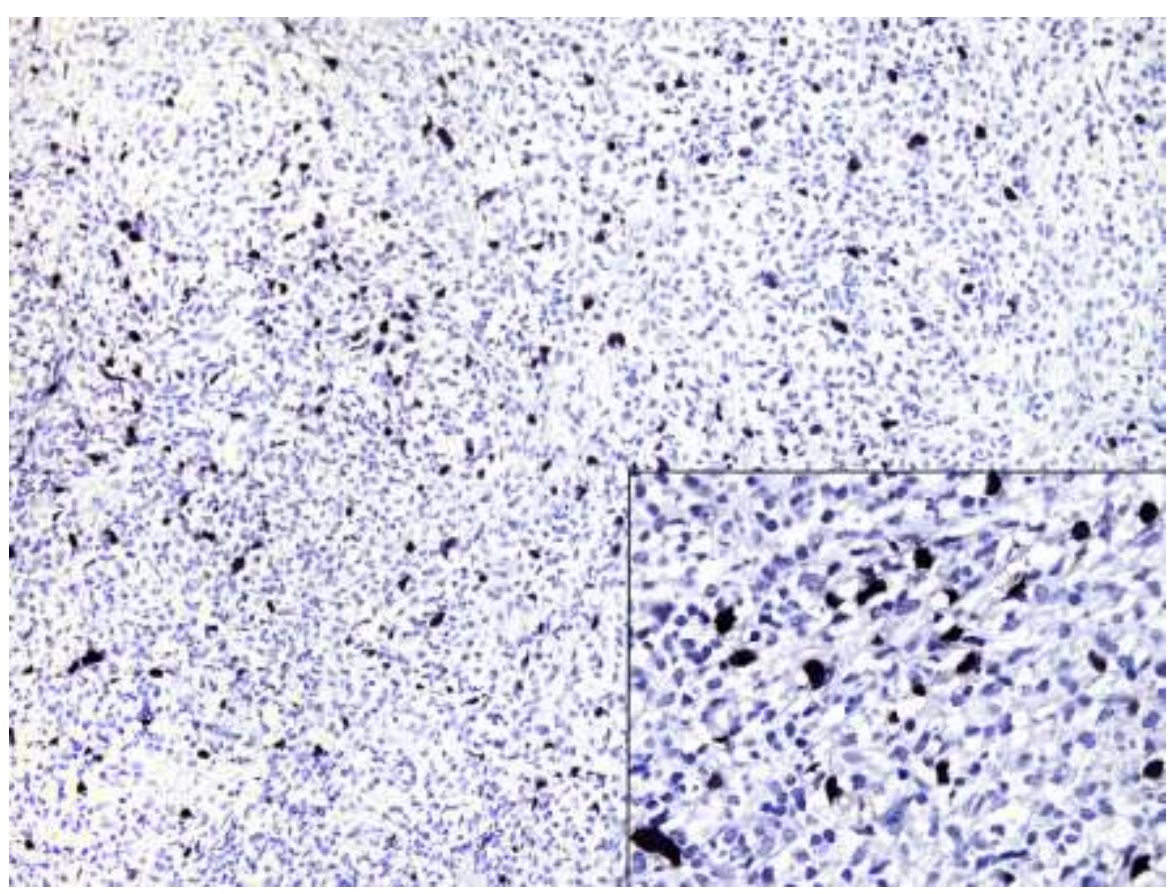

Fig. 10. EBER positivity detected in small, intermediate and larger B-cells (EBER-ISH, original magnification $\mathrm{x} 40$ ). Insert: Most of the large immunoblastic cells as well as the cells with RS-like morphology are positive for EBER (EBER-ISH, original magnification x200 - case two). A high-resolution version of this slide for use with the Virtual Microscope is available as eSlide: VM00711. 


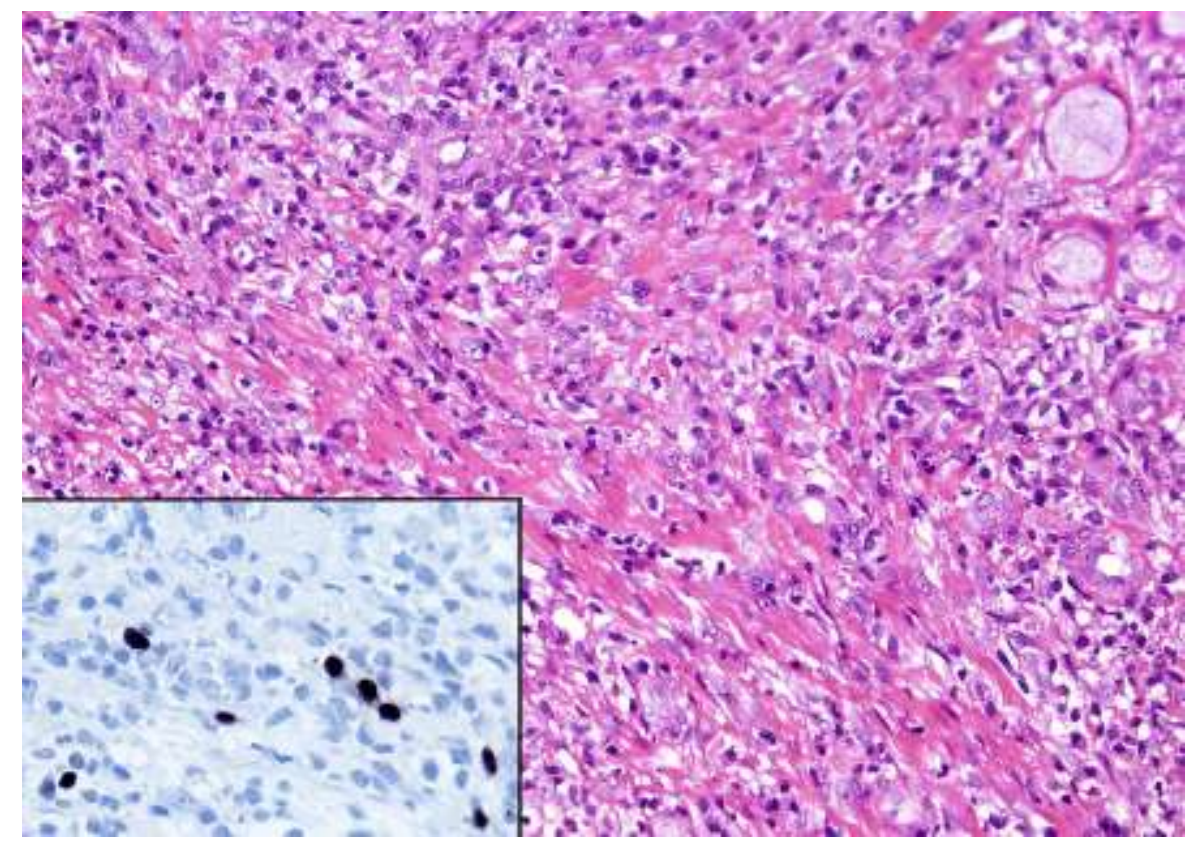

Fig. 11. Polymorphous nature of the infiltrate consisting of lymphocytes of various size with larger immunoblastic cells from case one (hematoxylin-eosin, original magnification x100). Insert: EBER positivity present in the infiltrate (EBER-ISH, original magnification x200).

\section{DISCUSSION}

Oral mucosal ulceration is a frequent clinical feature of HIV infection, the presence and severity of which may be an indication of immune status and disease progression. ${ }^{14}$ The diverse origin of ulcers in these patients is diagnostically challenging and may result in delayed therapeutic management. ${ }^{1}$ The ability to distinguish between the varied etiologies both clinically and histopathologically is thus essential.

EBV MCU represents a form of LPD which arises in the setting of defective cellular immunity. Previously published cases of EBV MCU of the oral mucosa arose in patients receiving immune suppressive medication and in the elderly with age-related immune senescence. ${ }^{8,9}$ The oropharynx is the most frequently involved mucosal site and is unique due to its close association with the lymphoid rich tissue of Waldeyers ring. The lymphotropic properties of EBV result in a concentration of latently infected B-lymphocytes at this site. 
Mucosal trauma and persistent antigenic stimulation may initiate activation of B-cells with uncontrolled B-cell proliferation which goes unchecked in cases of immunosuppression and where T-cell function is lost as seen in aging and in HIV infection. This complex interplay of defective host immunity in the face of persistent chronic inflammation is what triggers and sustains the proliferation of EBV-infected lymphocytes. ${ }^{12}$

The uncontrolled lymphoid hyperplasia comprises numerous activated B-cells and gives rise to a localized lymphoproliferative disorder which may ultimately culminate in true B-cell immortalization and transformation. ${ }^{9,12,13}$ The B-cell LPDs are a heterogeneous group of lesions which are classified by the World Health Organization (WHO) into early polyclonal lymphoid hyperplasias, polymorphous infiltrates and ultimately monomorphous B-cell proliferations with features of malignant lymphomas. ${ }^{11,12}$

EBV MCU presents with localized, solitary areas of mucosal ulceration. The ulcer base in EBV MCU is characterized by a superficial, well-circumscribed inflammatory infiltrate that is striking both for its density and polymorphous nature ${ }^{9}$. The infiltrate comprises a range of cell types with B-cell predominance. The B-lymphocytes range from being small to intermediate in size with scattered larger immunoblastic forms. Many of these cells are atypical and have a Reed-Sternberg (RS)-like morphology which may be of concern to the histopathologist. All forms of B-cells show expression of B-cell markers such as CD20 and CD79a, although this may be somewhat reduced. Interspersed CD3-positive small Tlymphocytes are seen throughout and have been noted to form a peripheral rim of cells in some cases, in an apparent attempt to wall off the hyperplastic lymphoid process. Fewer scattered histiocytes, eosinophils and neutrophils may be demonstrated. ${ }^{9}$

The B-cells show co-expression of CD30 in keeping with an activated phenotype. ${ }^{15}$ In addition, many of these cells are immunopositive for CD15. True RS cells also co-express 
both CD30 and CD15 but they lack expression of leukocyte common antigen as well as the usual B-cell markers as seen in EBV MCU. The entire range of B-cells in EBV MCU is positive for EBER confirming active viral intranuclear integration and EBV-induced lymphoid proliferation. Furthermore, the B-cells within these lesions demonstrate a postgerminal center phenotype as demonstrated by positivity for Mum-1 and negativity for the germinal center markers CD10 and Bcl-6.

Proliferative indices as demonstrated by nuclear Ki-67 immunolabeling are highly variable and non-contributory to the diagnosis. Nuclear signaling is identified in 10 to $50 \%$ of all cell types within the infiltrate. In addition, molecular testing for oligoclonal and monoclonal Bor T-cell populations is also not diagnostically significant. Gene rearrangement studies confirmed B-cell clonality in only $39 \%$ of the initial series of 26 cases of EBV MCU documented. At the same time, $38 \%$ of the lesions showed evidence of T-cell clonality due to the T-cell rich infiltrates often present. $^{9}$

EBV MCU needs to be distinguished from the monomorphic LPDs, which represent true high-grade neoplastic B-cell proliferations with clonal B-cell populations. These include the EBV-associated extra-nodal Classical Hodgkin Lymphoma (HL) and the EBV-associated diffuse large B-cell lymphoma (DLBCL) typically seen in elderly patients. Extra-nodal HL is exceedingly rare at oral mucosal sites and the neoplastic lesional cells are true RS cells. The RS cells express CD30, CD15 and are positive for EBER but do not co-express markers of Bcell lineage such as CD20. Although these lesions have a prominent mixed inflammatory cell infiltrate background, Ki-67 immunopositivity is generally restricted to the neoplastic RS cells.

DLBCL within the spectrum of LPDs is usually seen in elderly patients who have reduced age-related T-cell function. The tumor cells form a monomorphous population, expressing 
B-cell markers and very occasionally CD30. They lack the co-expression of CD15 as seen in HL and in EBV MCU. ${ }^{9,13}$ The immunophenotype described in EBV MCU applies to the full range of B-cells within these lesions whilst in HL, this is restricted to the neoplastic RS cells only. Furthermore, in contrast to true neoplastic proliferations, EBV DNA is usually not detected in peripheral blood studies in cases of EBV MCU. ${ }^{10}$

The very unusual benign form of chronic oral mucosal ulceration represented by the traumatic ulcerative granuloma with stromal eosinophilia (TUGSE) may bear some superficial clinical and histological resemblance to EBV MCU. TUGSE presents as a solitary chronic ulcer most often involving the tongue and more rarely at other intra-oral sites. A history of trauma is present in some but not all cases and the exact etiology of this form of ulceration is poorly understood. ${ }^{15-17}$ The ulcers are histopathologically characterized by a polymorphous inflammatory infiltrate, often dominated by an abundance of eosinophils. ${ }^{15}$ Although eosinophils are often recognized in EBV MCU, these are generally not as conspicuous. The infiltrate in TUGSE extends deep into the submucosa and contains numerous large atypical mononuclear cells which appear to be of T-cell phenotype. ${ }^{15,17}$ Early reports of this lesion indicated that some of these cells represented macrophages. ${ }^{18}$ The large atypical cells have been shown to co-express CD30, a feature of both activated B-and T-cells.

Both TUGSE and EBV MCU are characterized by alarming clinical and histological features, although they are indolent self-limiting forms of oral ulceration which may show spontaneous regression with uneventful healing. The distinct histopathological and immunophenotypic features should allow for easy distinction of these entities. ${ }^{9,15}$

Whilst lymphomatoid granulomatosis may also enter the differential diagnosis of an EBV MCU in the setting of immunosuppression, these lesions primarily present with pulmonary 
involvement. They are characterized by marked vasculitic changes and intraluminal thrombi in a T-cell rich background with scattered EBV-positive lymphoblastic cells. ${ }^{19}$ The lack of lung involvement and vasculitic changes may allow for distinction from EBV MCU.

Necrotizing ulcerative stomatitis (NUS) is a rapidly progressive ulceronecrotic condition arising de novo or progressing from necrotizing ulcerative gingivitis and periodontitis. It represents an opportunistic anaerobic infectious process largely limited to the soft tissue but which may spread to involve the adjacent bone causing widespread necrosis and destruction. ${ }^{6}$, 20 NUS may have overlapping clinical features with some of the other atypical ulcers. Histopathological examination of NUS shows numerous atypical macrophages which are highlighted by dense CD68 immunopositivity. The macrophages have irregular crescentic nuclei and can be demonstrated within the walls of intralesional blood vessels resulting in a histiocytic vasculitis. The ulcers are much deeper than those seen in EBV MCU and are associated with adjacent soft tissue necrosis, lacking the circumscription noted in EBV MCU. ${ }^{6}$

Most of our current treatment approaches have been inferred from the management of LPDs in post-transplant patients with iatrogenic immune suppression. The use of cytotoxic chemotherapeutic agents for EBV MCU is not recommended as it may compromise a patient's condition further by predisposing an individual who is already vulnerable at the outset to a higher risk of acquiring life threatening infection. ${ }^{12,13}$ The initiation of HAART appears to be the preferred treatment of choice for LPDs in HIV/AIDS ${ }^{11,12}$ as it may allow for recovery of immune function by decreasing HIV viral loads thereby allowing for increased $\mathrm{CD}^{+}{ }^{+}$-cell counts which restore some of the immune function.

Both patients presented here were therefore referred for initiation of appropriate antiretroviral therapy. Although the first patient had been receiving antiretroviral therapy (ART) for a year 
prior to presentation, he was, by his own admission, non-compliant. The resultant fluctuations in immune function predispose such a patient to infectious diseases and increase the risk of LPDs. It was suggested that the patients ART drug regimen be re-evaluated and re-initiated. The second patient had an astounding viral load and dismal $\mathrm{CD} 4^{+} \mathrm{T}$-cell count and had never received ART. The patient was referred for initiation of HAART to allow for immune reconstitution. Both patients were unfortunately lost to follow up after referral to local HIV clinics.

\section{CONCLUSION}

EBV MCU is a recently recognized form of LPD occurring in patients with immunosuppression. Its indolent clinical behavior, excellent long-term prognosis and unique constellation of clinical, and histomorphological features necessitate its distinction from other forms of ulceration. Oral ulcers occurring in HIV infected patients pose a diagnostic dilemma due to the multitude of potential etiologies in this patient group. EBV MCU should be included in the differential diagnosis of oral ulceration in HIV/AIDS. The importance of a detailed clinical and medical history cannot be overemphasized in the diagnosis of these lesions. It is imperative that chronic non-healing ulcers in HIV/AIDS patients be biopsied in order for an accurate diagnosis to be rendered and for the most appropriate therapy to be instituted.

\section{REFERENCES}

1 Ficarra G. Oral ulcers in HIV-infected patients: an update on epidemiology and diagnosis. Oral Dis. 1997;3 Suppl 1:S183-189.

2 Delgado WA, Almeida OP, Vargas PA, Leon JE. Oral ulcers in HIV-positive Peruvian patients: an immunohistochemical and in situ hybridization study. J Oral Pathol Med. 2009;38:120-125. 

Oral Pathol. 1992;73:212-214. Classification and diagnostic criteria for oral lesions in HIV infection. EC-Clearinghouse on Oral Problems Related to HIV Infection and WHO Collaborating Centre on Oral Manifestations of the Immunodeficiency Virus. J Oral Pathol Med. 1993;22:289-291. Jones AC, Gulley ML, Freedman PD. Necrotizing ulcerative stomatitis in human immunodeficiency virus-seropositive individuals: a review of the histopathologic, immunohistochemical, and virologic characteristics of 18 cases. Oral Surg Oral Med Oral Pathol Oral Radiol Endod. 2000;89:323-332. Piluso S, Ficarra G, Lucatorto FM, Orsi A, Dionisio D, Stendardi L, et al. Cause of oral ulcers in HIV-infected patients: a study of 19 cases. Oral Surg Oral Med Oral Pathol Oral Radiol Endod. 1996;82:166-172.

8 Attard AA, Praveen P, Dunn PJ, James GJ. Epstein-Barr virus-positive mucocutaneous ulcer of the oral cavity: the importance of having a detailed clinical history to reach a correct diagnosis. Oral Surg Oral Med Oral Pathol Oral Radiol. 2012;114:e37-39.

9 Dojcinov SD, Venkataraman G, Raffeld M, Pittaluga S, Jaffe ES. EBV positive mucocutaneous ulcer--a study of 26 cases associated with various sources of immunosuppression. Am J Surg Pathol. 2010;34:405-417.

10 Hart M, Thakral B, Yohe S, Balfour HH, Jr., Singh C, Spears M, et al. EBV-positive Mucocutaneous Ulcer in Organ Transplant Recipients: A Localized Indolent Posttransplant Lymphoproliferative Disorder. Am J Surg Pathol. 2014.

11 Swerdlow SH CE, Harris NL, Jaffe ES, Pileri SA, Stein H, et al. WHO classification of tumours of haematopoietic and lymphoid tissues. Lyon: IARC press: 2008.

12 Fujita H, Nishikori M, Takaori-Kondo A, Yoshinaga N, Ohara Y, Ishikawa T, et al. A case of HIV-associated lymphoproliferative disease that was successfully treated with highly active antiretroviral therapy. Int J Hematol. 2010;91:692-698. 
13 Mahe E, Ross C, Sur M. Lymphoproliferative Lesions in the Setting of HIV Infection: A Five-Year Retrospective Case Series and Review. Patholog Res Int. 2011;2011:618760.

14 Bodhade AS, Ganvir SM, Hazarey VK. Oral manifestations of HIV infection and their correlation with CD4 count. J Oral Sci. 2011;53:203-211.

15 Hirshberg A, Amariglio N, Akrish S, Yahalom R, Rosenbaum H, Okon E, et al. Traumatic ulcerative granuloma with stromal eosinophilia: a reactive lesion of the oral mucosa. Am J Clin Pathol. 2006;126:522-529.

16 Gagari E, Stathopoulos P, Katsambas A, Avgerinou G. Traumatic ulcerative granuloma with stromal eosinophilia: a lesion with alarming histopathologic presentation and benign clinical course. Am J Dermatopathol. 2011;33:192-194.

17 Salisbury CL, Budnick SD, Li S. T-cell receptor gene rearrangement and CD30 immunoreactivity in traumatic ulcerative granuloma with stromal eosinophilia of the oral cavity. Am J Clin Pathol. 2009;132:722-727.

18 Eversole LR, Leider AS, Jacobsen PL, Kidd PM. Atypical histiocytic granuloma. Light microscopic, ultrastructural, and histochemical findings in an unusual pseudomalignant reactive lesion of the oral cavity. Cancer. 1985;55:1722-1729.

19 Jaffe ES. Lymphoid lesions of the head and neck: a model of lymphocyte homing and lymphomagenesis. Mod Pathol. 2002;15:255-263.

20 Williams CA, Winkler JR, Grassi M, Murray PA. HIV-associated periodontitis complicated by necrotizing stomatitis. Oral Surg Oral Med Oral Pathol. 1990;69:351-355. 